\title{
Las Tareas del Líder Inclusivo en Centros Educativos de Zonas Desfavorecidas y Favorecidas
}

\author{
The Task of the Inclusive Leader in Educational Centers in \\ Disadvantaged and Favored Areas
}

\author{
M José León * \\ Emilio Crisol \\ Rubén Moreno Arrebola \\ Universidad de Granada, España
}

\begin{abstract}
El liderazgo participativo, democrático y transformacional del director ejerce una influencia positiva en la práctica del profesorado y en la calidad de los aprendizajes y participación de todos los estudiantes, así como en la mejora de los centros y en la creación de una cultura inclusiva. Se realiza un cuantitativo ex-post facto, de tipo transversal cuyo objeto es conocer las tareas de la dirección que promueven la inclusión y determinar qué características, de los directores y los centros, correlacionan positivamente. En este artículo, se presenta los resultados del cuestionario: Liderando la Educación Inclusiva (LEI-Q) aplicado a 397 miembros de familias y equipos docentes de 17 centros educativos. En análisis de datos es de tipo descriptivo e inferencial, mediante la prueba no paramétrica U Man-Whitney. Los resultados muestran que los miembros de la comunidad educativa (equipo docente y familias) de todos los centros participantes consideran que sus equipos directivos han implantado "sustancialmente" la mayor parte de las acciones que contribuyen a que sus centros sean inclusivos. Sin embargo, los equipos directivos de centros situados en contextos favorecidos lo han hecho mayormente que aquellos que se encuentran en contextos desfavorecidos y, de forma más significativa, en las acciones pertenecientes a comunidad profesional de aprendizaje y gestionar procesos de enseñanza-aprendizaje.
\end{abstract}

Descriptores: Educación intercultural; Liderazgo; Escuela desfavorecida; Administrador de la educación.

The participative, democratic and transformational leadership of the director exerts a positive influence on the practice of the teaching staff and on the quality of the learning and participation of all the students, as well as on the improvement of the centers and the creation of an inclusive culture. An ex-post facto quantitative, cross-sectional type is carried out whose purpose is to know the management tasks that promote inclusion and determine which characteristics, of the directors and centers, correlate positively. In this article, we present the results of the questionnaire: Leading Inclusive Education (LEI-Q) applied to 397 family members and teaching teams from 17 educational centers. In data analysis, it is descriptive and inferential, using the non-parametric U Man-Whitney test. The results show that the members of the educational community (teaching staff and families) of all the participating centers consider that their management teams have implemented "substantially" most of the actions that contribute to their centers being inclusive. However, the management teams of centers located in favored contexts have done so more than those in disadvantaged contexts and, more significantly, in the actions pertaining to the professional learning community and managing teachinglearning processes.

Keywords: Inclusive education; Leadership; Disadvantaged schools; Educational administrators.

*Contacto: mleon@ugr.es

ISSN: $1696-4713$

www.rinace.net/reice/

revistas.uam.es/reice
Recibido:

30 de noviembre 2017

$1^{\text {a }}$ Evaluación: 27 de diciembre 2017

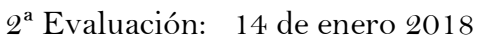

Aceptado: $\quad 2$ de febrero 2018 


\section{Introducción}

Existe un creciente interés en el estudio del liderazgo escolar por su repercusión en la práctica y en la política de los sistemas y centros educativos inclusivos. Muestra de ello es el gran número de trabajos que se vienen desarrollando sobre este aspecto (Angelides, 2012; Angelides, Antoniou y Charalambous, 2010; Blandford, 2013; León, 2001, 2012; León et al., 2016; Ryan, 2006), así como la publicación en los últimos años de informes a nivel de Unión Europea que están influyendo en la política educativa de los países y de los centros educativos que conforman la misma, tales como el publicado por la OCDE "Mejorar el liderazgo escolar" (Pont, Nusche y Moorman, 2008), "Hacia un marco de referencia en el liderazgo escolar" (Bolhöfer, 2011), "Conclusiones del Consejo sobre liderazgo educativo eficaz" (Diario oficial de la Unión Europea, 2014). Sin embargo, es llamativo que en ninguno de ellos se aborde adecuadamente los desafíos relacionados con la inclusión escolar, aspecto este que, tanto a nivel político (una revisión de los países europeos en los que la inclusión es parte importante en la política educativa de los mismos se puede encontrar en Precey y Mazurkiewcz, 2013) como de investigación (Arnáiz, Azorín y García, 2015; Dyson, Howes y Roberts, 2002; Riehl, 2000), se ha constituido en un objetivo prioritario de la mayor parte de los sistemas educativos basados en el convencimiento de que el modelo de escuela hacia el que deben tender actualmente los centros es el inclusivo, y que para su consecución un elemento clave es el liderazgo escolar. Por ello consideramos de gran relevancia profundizar en el grado en que la dirección de los centros educativos lleva a cabo tareas que promueven la inclusión y determinar qué características, tanto de los directores, como de los centros, correlacionan positivamente con las mismas.

\section{Fundamentación teórica}

Según destacan Macmillan y Edmunds (2010), hay evidencias significativas de que el éxito de los programas de inclusión es atribuible, en gran parte, al liderazgo ejercido por el director y a su capacidad para crear una escuela inclusiva. En este sentido, si bien el análisis de los centros educativos exitosos e inclusivos, evidencia que en ellos no se da una sola forma de llevar a cabo el liderazgo (Gómez-Hurtado y Ainscow, 2014; Pamies, Senent y Essomba, 2016), la puesta en práctica de un liderazgo participativo, distribuido, democrático y transformacional y dirigido a crear cultura inclusiva, se ha postulado como el más adecuado (Blandford, 2013; Murillo, 2006; Murillo y Hernández-Castilla, 2014; Ryan, 2006).

Este tipo de liderazgo esta contextualizado, se muestra comprometido con la diferencia (Gunter, 2006) y es crítico con las formas de escolarización homogéneas que se han mostrado inapropiadas para alcanzar una educación de calidad para todos y todas, sobre todo para aquellas personas más vulnerable (Mac Ruairc, 2013). Por ello, está surgiendo con fuerza un cuerpo considerable de trabajos que se centran en la necesidad de que los equipos directivos critiquen los modelos actuales de escolarización y aborden un liderazgo centrado en valores como la equidad y la justicia social a través de la construcción y fortalecimiento de una comunidad democrática en la escuela, ya que, como afirma Fraser y Shields (2010) "las aulas son un microcosmos de la sociedad y refleja las normas dominantes, el poder, los privilegios y el estado, a menos que los 
líderes escolares diseñen y promulguen políticas específicas para garantizar la justicia social" (p. 13).

El liderazgo inclusivo ejerce una influencia positiva en la práctica del profesorado (Angelides, Antoniu y Chalambous, 2010), en la calidad de los aprendizajes y participación de los estudiantes (Ainscow y Kaplan, 2005), así como en la mejora de los centros y en la creación una cultura inclusiva (Muijs, 2006; Ryan, 2006). Por ello, es necesario profundizar en las características y prácticas que desempeñan los equipos directivos para alcanzar estas metas, en la formación necesaria para ejercerlas y en las condiciones/factores que confluyen para que se desempeñen adecuadamente las mismas.

Algunas características centrales del liderazgo inclusivo o del liderazgo educativo inclusivo, como lo denomina Dorczak (2013, p. 53), son:

- Valora el proceso social y el trabajo en equipo como los elementos principales del contexto de desarrollo personal y organizacional.

- Involucra activamente a todos según su potencial.

- Permite que todos escuchen (y valoren) su voz y actúen, creando las condiciones para un buen trato interpersonal y una comunicación profesional.

- Les da a todos el espacio y la posibilidad de desarrollarse personalmente y profesionalmente dentro del grupo.

- Aplica la regla de un cambio y desarrollo continuo de individuos, grupos y estructuras organizacionales.

- Se basa en valores como la autonomía, el respeto mutuo, la confianza, el cuidado y la responsabilidad para otros en el grupo y en un sentido más amplio.

- Se ve como un proceso de desarrollo de poder distribuido (no empoderamiento visto como el resultado de una acción controlada por el líder, sino de empoderamiento donde las personas asumen gradualmente responsabilidades a medida que se desarrollan).

De igual modo, según Causton y Theotaris (2017, p. 3), los directores que tienen éxito en liderar escuelas plenamente inclusivas, son aquellos que:

- Establecen una visión audaz y clara de la plena inclusión, basada en la idea de que todos los niños pueden aprender.

- Participan en la planificación e implementación colaborativa con el personal.

- Desarrollan y apoya a los equipos de profesionales.

- Reducen la fragmentación de iniciativas.

Estas tareas son similares a las señaladas en el informe "Hacia un marco de referencia de en el liderazgo escolar" (Bolhöfer, 2011), en el cual queda reflejado cinco ámbitos destacados por los socios europeos como importantes para el liderazgo escolar en sus países:

- Expectativas políticas y culturales y su traducción a significado y dirección internos.

- Comprender y otorgar poderes al profesorado y otro personal. 
- Culturizar y estructurar centros educativos.

- Trabajar con socios y con el entorno.

- Crecimiento y desarrollo personal (Dorczak, 2013, p. 7).

De acuerdo con esto, y con las conclusiones extraídas de investigaciones relevantes sobre el liderazgo inclusivo (Angelides, Antoniu y Charalambous 2010; Baker, 2007; Blandford, 2013; Macmillan, 2010; Ryan, 2006), las tareas y/o funciones desempeñadas por el equipo directivo para cumplir las metas de un centro inclusivo han sido agrupadas por nosotros en estudios anteriores (León, 2001, 2012; León et al., 2016) en: i) Apertura a la comunidad, que implica llevar a cabo iniciativas desde el centro educativo que potencian la apertura del centro hacia al entorno y la familia; ii) El Centro como comunidad inclusiva, para lo que el equipo directivo emprende acciones para generar una visión compartida, promoviendo la participación, cooperación y dinámicas de reflexión positiva de la comunidad educativa hacia la diversidad; iii) Comunidad Profesional de Aprendizaje, el líder inclusivo promueve la formación, el desarrollo profesional del profesorado y la creación de comunidades profesionales de aprendizaje; y iv) Gestión de los procesos de enseñanza-aprendizaje, llevando a cabo iniciativas para mejorar y favorecer la coordinación en el proceso de enseñanza y aprendizaje del profesorado.

Los centros educativos deben estar abiertos a la comunidad, por lo que los lideres escolares deben conocer y responder a las necesidades de la misma para hacerlas efectivas, adaptándolas y traduciéndolas a todos los miembros del centro educativo, para que ellas formen parte de la misión del centro. Esto, según Bolhöfer (2011) "coloca a los líderes de los centros en una posición en la que necesitan interpretar, traducir y dilucidar las demandas externas para facilitar el entendimiento y crear un sentido compartido de dirección en sus centros" (p. 8).

Por otro lado, las acciones dirigidas a conseguir que el centro se constituya en una comunidad inclusiva conllevan, por parte del equipo directivo, la creación, de manera colaborativa y participativa con el resto de personas que forman parte del centro, de creencias, actitudes, normas y comportamientos acordes con los valores de equidad y justicia social. En este sentido, Specht y Young (2010) muestran algunas estrategias que permiten crear una comunidad escolar inclusiva:

- Creando ambientes de apoyo para promover una atmosfera de bienvenida y una cultura escolar que acepta la diversidad.

- Desarrollando relaciones que sean modelos a seguir por los estudiantes.

- Propiciando la participación.

- Promoviendo competencias en el profesorado y en la comunidad escolar.

- Creando vínculos entre la casa y la escuela.

De igual modo, los líderes escolares han de crear una cultura organizativa basada en estructuras que armonicen con ella. En este sentido, y de acuerdo con la tipologías organizacionales descritas por Handy (1985, citado por Dorczak, 2013): Zeus, Apolo, Ateniense y Dionisiaca, la cultura organizativa de los centros inclusivos responde a la llamada cultura ateniense, ya que, según Dorczak (2013), se basa en el trabajo en equipo, crea las condiciones necesarias para que se produzca la comunicación interpersonal y profesional y la convierte en la herramienta principal para resolver problemas con los 
que se puede enfrentar la organización. Asimismo, intenta darse cuenta del potencial de todos los miembros de la organización, valora la creatividad de todos y crea las condiciones para el desarrollo profesional y personal, da pautas para estos y hace que la creatividad individual sea parte del esfuerzo colaborativo.

Por todo ello, sin duda, este tipo de cultura organizativa es la mejor posible para las instituciones educativas, ya que brinda buenas condiciones para la cooperación y crea sinergias de trabajo colaborativo. De acuerdo con Dorczak (2013), este tipo de cultura organizativa, evidentemente, se encuentra relacionada con el estilo de liderazgo inclusivo.

Hay pocas dudas de que es la mejor cultura organizacional, de entre los cuatro tipos señalador por Handy con el propósito de desarrollar un liderazgo educativo inclusivo y educación inclusiva en las escuelas. La cultura organizacional ateniense es un buen contexto para el liderazgo transaccional y, a veces, el liderazgo transformacional porque implica colaboración en equipo que generar sinergias y nuevas ideas para el desarrollo escolar. (p. 52)

Otra función que deben desempeñar los lideres escolares es la de fomentar el desarrollo de las competencias del profesorado, facilitando las condiciones adecuadas y creando estructuras que les lleven a trabajar en equipo y a que se produzca una distribución del liderazgo, propiciando que sea el propio profesorado el que lidere la mejora. El centro, por tanto, se constituye en una comunidad profesional de aprendizaje que promueve el desarrollo profesional y de todas las personas que lo forman, por lo que el principal objetivo del equipo directivo es diseñar estructuras y culturas de centro que apoyen la capacidad de construir y aprender de un docente para mejorar y favorecer la coordinación en el proceso de enseñanza y aprendizaje del profesorado. En este sentido, Dorczak (2013) señala,
el papel principal del líder escolar es reconocer y utilizar los procesos sociales de aprendizaje organizacional e individual para liberar y desarrollar los talentos de todos los profesores u otros miembros del personal, así como reconocer y activar el potencial de todos los estudiantes que son materia principal y valor básico en el trabajo escolar. (p. 55)

Por último, dentro de las tareas desempeñadas en los centros inclusivos, los líderes escolares deben gestionar la mejora del proceso de enseñanza-aprendizaje y su evaluación creando estructuras que permitan que el profesorado reflexione de forma conjunta sobre la práctica e intercambien su conocimiento, ya que "los directores tienen un impacto indirecto en el aprendizaje de los estudiantes, pero juegan un papel muy importante en relación a las condiciones que aumentan ese aprendizaje" (Causton y Theotaris, 2017, p. 3)

El profesorado del centro, incluidos los especialistas en atención a la diversidad, tienen como meta mejorar el aprendizaje de todo el alumnado, y la mejor forma es creando equipos de trabajo (equipos instructivos) formados por el profesor de atención a la diversidad, el generalista, para-profesionales, familia, terapeuta ocupacional, fisioterapeuta, logopedas o terapeutas del habla y el lenguaje, psicólogos, trabajadores sociales y profesores especialistas en visión y audición.

El equipo directivo será el encargado de crear y dinamizar el trabajo de estos equipos. Causton y Theotaris (2017) indican al respecto que serán los encargados de establecer y supervisar las expectativas educativas inclusivas, explicándolas al equipo en términos de coplanificaión y coenseñanza, de dotar de tiempo a los equipos para la planificación 
(como mínimo de 40 a 60 minutos una o dos veces por semana), de propiciar el desarrollo profesional sobre estrategias de colaboración y coenseñanza y de conseguir que cada uno conozca al otro desde el punto de vista personal y profesional.

Por otro lado, el análisis de los centros educativos en los que se llevan a cabo culturas, políticas y prácticas inclusivas, nos ofrece información sobre cuáles son las condiciones/factores de los centros y su entorno que les permiten mejorar los centros y promover la inclusión. En este sentido, además del tipo de liderazgo ejercido, el cual debe ser distribuido y transformacional (Angelides, 2012; Blandford, 2013), algunos de los factores que condicionan la eficacia de este equipo son: la forma de acceder al cargo (Gómez-Hurtado y Ainscow, 2014), el tiempo de permanencia en el mismo (Sindelar et al., 2006), la formación recibida sobre atención a la diversidad e inclusión (Conrad y Brown, 2011; León, 2012), las actitudes y el compromiso con valores relacionados con la justicia y la equidad (Gómez-Hurtado y Ainscow, 2014) y la participación en innovaciones educativas (Klingner et al., 2001). Todo esto es posible si la autonomía otorgada a los centros y a sus miembros es realmente empoderante y les permite tomar decisiones significativas (OCDE, 2009). Es entonces cuando hay realmente posibilidades de que los lideres escolares se vean inmersos y fomenten innovaciones educativas (Leithwood y Prestine, 2002; Mac Ruairc, 2013) alineadas con los valores de equidad, justicia social e inclusión a fin de que las mejoras del centro permitan mayor grado de igualdad.

La mejora de las competencias profesionales de los equipos directivos en relación a la inclusión y a la atención a la diversidad, no sólo incide positivamente en el profesorado, sino que, como afirma Bolívar (2010), es un factor indirecto que contribuye al aprendizaje de todo el alumnado. Así, el análisis de las buenas prácticas desarrolladas por los directivos en contextos inclusivos, unido al centrado en los programas formativos dirigidos a los mismos, nos han permitido destacar los siguientes conocimientos /actitudes/ habilidades como la base de los programas de formación dirigidos a los líderes para que su ejercicio sea eficaz e inclusivo (León y López, 2017): a) Liderazgo distribuido, democrático, inclusivo; b) Misión, visión y valores inclusivos y de justicia social; c) Ética y normas profesionales; d) Estrategias de planificación y enseñanza colaborativa; e) Comunicación, cooperación y colaboración; f) Equidad y prácticas culturalmente sensibles; g) Conocimiento y apertura a la comunidad y compromiso de las familias; h) Comunidad profesional de aprendizaje de profesores y personal; i) Comunidad de cuidado y apoyo al alumnado; j) Gestión y evaluación de procesos de enseñanza-aprendizaje; k) Gestión y administración de los recursos escolares (personales y materiales); 1) Gestión de la calidad, investigación, reflexión en y sobre la práctica y mejora escolar.

Por otro lado, y por lo que respecta al centro educativo y su entorno, la participación con otros centros e instituciones (Ainscow y Howes, 2007) y de la comunidad educativa del centro (Gómez-Hurtado y Ainscow, 2014), las buenas relaciones e interacciones que producen análisis y reflexión entre todos los miembros del centro, incluidos los niños y niñas (Ainscow y Kaplan, 2005; Angelides, 2012; Dyson, 2006; Gómez-Hurtado y Ainscow, 2014), el apoyo prestado por el personal especializado en educación especial y atención a la diversidad (Gómez-Hurtado y Ainscow, 2014) y la zona en la que se encuentra la escuela, pueden ser factores diferenciadores en cuanto a la manera de ejercer la dirección y del grado en que ésta promueve la inclusión. 
En este sentido, y con respecto a este último aspecto, que es en el que hemos centrado parte del estudio desarrollado, algunos investigadores (Sammons y Bakunn, 2011) concluyen que cuando las escuelas son exitosas, no existen características diferenciales en la dirección, aunque éstos se encuentren en contextos desfavorecidos. Por su parte, en el trabajo realizado por Gómez-Hurtado y Ainscow (2014) con tres escuelas urbanas de zonas desfavorecidas de Manchester, los autores concluyen que, si bien existen diferencias en relación al estilo directivo ejercido (dos directores poseen un estilo democrático/distribuido y uno autoritario), los tres defienden prácticas directivas inclusivas. A resultados similares llegan Iranzo, Tierno y Barrio (2014), quienes concluyen que los modelos de liderazgo desarrollados por los equipos directivos de estas zonas son distribuidos y contextualizados, al igual que en los centros de zonas favorecidas.

La mejora de las escuelas es un objetivo prioritario, por lo que los equipos directivos de las mismas, los cuales deben desarrollar acciones encaminadas a mejorar los aprendizajes de todo el alumnado y poner en acción valores inclusivos, entre los que, según Ainscow y Both (2015) se destacan, por contribuir más que los demás en el desarrollo de la inclusión, la igualdad, participación, comunidad, respeto a la diversidad y sostenibilidad.

La revisión en base de datos educativas de relevancia, llevada a cabo por Muijs (2003) muestra que, entre los principales factores que conducen a la mejora y eficacia de las escuelas situadas en zonas desfavorecidas están el liderazgo eficaz, junto a que se centran en la enseñanza aprendizaje, implican a padres, se convierten en comunidades de aprendizaje, enfatizan el continuo desarrollo profesional, crean un entorno rico en información, y una cultura escolar positiva, existe apoyo externo e incorporan programas de mejora.

En esta misma línea, la investigación desarrollada por Pamies, Senent y Essomba (2016) señalan al liderazgo pedagógico ejercido por los equipos directivos como uno de los principales factores que contribuyen al éxito académico y a la continuidad educativa de los jóvenes de centros educativos situados en entornos desfavorecidos y destacan que este liderazgo incide en estos contextos, en la aceptación positiva de los jóvenes escolarizados, en la búsqueda constante de soluciones para los retos que se plantean de forma cotidiana en el centro, en la elaboración de un proyecto de centro compartido que asegura la adquisición de las competencias a todo el alumnado, en el impulso a la participación en programas y proyectos educativos y establecimiento de redes de apoyo con el fin de adecuar y enriquecer la respuesta educativa del centro, en la comunicación, coordinación y colaboración con los centros educativos del entorno, en el establecimiento de estructuras que permitan, desde la colaboración con otras instancias, contribuir a dar respuesta adecuada a las necesidades de todos los alumnos, desde una perspectiva inclusiva, en el desarrollo de un clima y una cultura escolar positiva, en la flexibilidad en la interpretación de las normas de la administración educativa y su defensa ante instancias superiores y en el desarrollo de estrategias de formación del profesorado ad-hoc sostenidas en el tiempo.

\section{Método}

El propósito general de la investigación ha sido conocer, según la opinión de los equipos docentes (profesorado y cargos directivos) y familias, la forma en que la dirección de 
centros de Granada lleva a cabo tareas que promueven la inclusión, así como determinar qué características del director y de los centros, correlacionan positivamente con las mismas. De entre estas últimas, nos centramos en este artículo en determinar en qué medida influye la zona (favorecida o desfavorecida) en la que se encuentra el centro educativo en las funciones que el equipo directivo lleva a cabo.

En cuanto al diseño de investigación, se trata de un estudio cuantitativo ex-post facto, de tipo transversal en el que, en un principio, se llevó a cabo un muestreo aleatorio estratificado con un cálculo del tamaño maestral que tenía en cuenta una proporción esperada del 66\% (basada en datos de pilotaje previo) y una precisión del $5 \%$. Estableciéndose la muestra necesaria para su significatividad en 101 centros educativos de Granada capital (37 centros privados, 64 centros públicos, 26 de educación primaria y 16 de secundaria. Sin embargo, aunque todos fueron invitados a responder el cuestionario, finalmente respondieron 17 de los cuales 7 son de Educación Primaria, 5 de Secundaria y 5 de Educación Primaria y Secundaria, 11 son públicos y 6 privados/concertados. Por lo que, al final se trata de un muestreo de carácter intencional en el que se ha tenido en cuenta que haya representación de todos los estratos.

Los miembros del equipo docente de los centros que han respondido al cuestionario han sido $243(58,1 \%)$, mientras que $154(41,9 \%)$ son miembros de las familias, de los cuales 198 son mujeres $(61,9 \%)$ y 121 hombres $(37,8 \%)$ pertenecientes a centros públicos (206 participantes de 11 centros) y 126, de 6 centros concertados. De los 396 participantes, 291 pertenecen a centros de zonas favorecidas (con un nivel socioeconómico medio-alto) y 106 a desfavorecidas (nivel socioeconómico bajo).

Como variables independientes se contemplan la zona socio-demográficas en la que está situado el centro educativo, diferenciándose entre zonas favorecidas y zonas desfavorecidas. Entendiendo por centros educativos de zonas favorecidas, aquellos en dónde el nivel socio-económico y socio-educativo de las familias y del alumnado del mismo es medio y/o alto (determinado por los directores/as de dichos centros), y por centros educativos de zonas desfavorecidas, aquellos en dónde el nivel socio-económico y socio-educativo es bajo. Como variables dependientes se contemplan las cuatro dimensiones que componen el "Cuestionario: Liderando la Educación Inclusiva" (LEI-Q) (León et al., 2016), el cual ha sido aplicado de manera presencial en los centros educativos, tras un primer contacto telefónico con el equipo directivo.

El cuestionario posee validez de contenido y fiabilidad (Alfa de Cronbach total: 0,981; Dimensión I: 0,966; Dimensión II: 0,963; Dimensión III: 0,958 y Dimensión IV: 0,956), es de tipo Likert, con cuatro opciones de respuesta (1. No implantado; 2. Parcialmente implantado; 3. Sustancialmente implantado y 4. Totalmente implantado), y está compuesto por 66 ítems, distribuidos en cuatro dimensiones: I. Apertura a la comunidad; II. Centro como comunidad inclusiva; III. Comunidad profesional de aprendizaje y IV. Gestionar procesos enseñanza-aprendizaje.

De las cuatro dimensiones que lo componen, los miembros de las familias sólo contestaron a las relativas a: Apertura a la comunidad y Centro como comunidad inclusiva (Dimensiones I y II), mientras que los miembros del equipo docente, también dieron respuesta a los ítems relacionados con: Comunidad profesional de aprendizaje y Gestionar procesos de enseñanza-aprendizaje (Dimensiones III y IV). 
Se ha llevado a cabo un análisis descriptivo y diferencial de los datos recogidos por medio del programa SPSS 24. En el análisis descriptivo se han obtenido las medias de las dimensiones en general, así como medias y desviación estándar de cada ítem del cuestionario. Para el análisis inferencial, se ha llevado a cabo la prueba no paramétrica $\mathrm{U}$ Man-Whitney, pues no se han obtienen buenos resultados en las pruebas de normalidad y homocedasticidad.

\section{Resultados}

Los resultados del análisis descriptivo por dimensiones se muestran en el cuadro 1, en la cual se puede apreciar que, de manera general, para el total de la muestra de los centros educativos analizados, las acciones del equipo directivo que contribuyen a la inclusión están "sustancialmente implantadas", ya que sus medias oscilan entre 3,03 (Dimensión I: Apertura a la comunidad) y 3,46 (Dimensión IV: Gestionar el proceso de enseñanzaaprendizaje) aunque, en todos los casos, lo están más implantadas en los centros situados en contextos favorecidos que desfavorecidos. En el caso de los equipos de zonas desfavorecidas, incluso, han implantado sólo de forma "parcial" las acciones relativas a la Apertura a la comunidad.

Cuadro 1. Puntuaciones medias en cada dimensión según el contexto

\begin{tabular}{lccc}
\hline & Total & ConteXto DESFAVORABLE & ConTEXTO FAVORABLE \\
\hline Dimensión 1 & 3,03 & 2,98 & 3,05 \\
Dimensión 2 & 3,21 & 3,16 & 3,22 \\
Dimensión 3 & 3,20 & 3,03 & 3,29 \\
Dimensión 4 & 3,46 & 3,29 & 3,55 \\
Total & 3,29 & 3,14 & 3,37 \\
\hline
\end{tabular}

Fuente: Elaboración propia.

$\mathrm{El}$ análisis por ítems de la respuesta dada por la muestra de equipos docentes y familias de centros pertenecientes a zonas desfavorecidas, con respecto a las acciones más abordadas por los equipos directivos de zonas favorecidas, en relación a la Apertura a la comunidad (cuadro 2), se distinguen porque las medias en este último contexto siempre son más altas, salvo en las tres acciones que ambos equipos directivos han abordado de forma "parcial": promover la colaboración con el mundo empresarial de manera continuada para reforzar la relación escuela-entorno laboral (ítem 3: 2,54 y 2,25 de media en centros desfavorables y favorables, respectivamente), organizar debates abiertos a la comunidad sobre situaciones de exclusión (racismo, xenofobia, machismo, ...) (ítem 6: 2,72 y 2,65) e impulsar acciones para colaborar con otros centros educativos, conocer y compartir experiencias (ítem 4: 2,78 y 2,71). Sin embargo, en ambos contextos, el equipo directivo viene aplicando de forma "sustancial" las acciones centradas en: escucha y tiene en cuenta las demandas y necesidades de todas las familias (ítem 13: 3,31 y 3,37 de media en centros desfavorables y favorables, respectivamente), ofrece las instalaciones del centro y sus recursos para el desarrollo de actividades (culturales, educativas,...) de interés para la comunidad (ítem 8: 3,28 y 3,32) y propone actividades educativas fuera del centro (ítem 5: 3,19 y 3,34).

Por lo que respecta a la dimensión del Centro como comunidad inclusiva (cuadro 3), de nuevo, las medias de las acciones desarrolladas por los equipos directivos son mayores en los centros de zonas favorecidas, salvo en tres ítems, de los 20 que forman esta 
dimensión: ítem 25. Desarrolla programas educativos para prevenir actitudes discriminatorias entre el alumnado; ítem 30. Promueve que los distintos miembros de la comunidad educativa participen en la evaluación de las labores de dirección e ítem 32. Establece mecanismos para impulsar la participación del alumnado en la regulación de conflictos que surgen en el entorno educativo

Cuadro 2. Puntuaciones medias y desviación estándar de las respuestas por ítem a la Dimensión I: Apertura a la comunidad

\begin{tabular}{lcccc}
\hline & \multicolumn{2}{c}{ ConTEXTo DeSFAvoRABLE } & \multicolumn{2}{c}{ ConTEXTO FAvORABLE } \\
\cline { 2 - 5 } & Medias & Desviación estándar & Medias & Desviación estándar \\
\hline Ítem 1 & 3,14 & 0,888 & 3,26 & 0,800 \\
Ítem 2 & 3,04 & 0,945 & 3,17 & 0,872 \\
Ítem 3 & 2,54 & 1,266 & 2,25 & 1,133 \\
Ítem 4 & 2,78 & 1,014 & 2,71 & 1,076 \\
Ítem 5 & 3,19 & 0,977 & 3,34 & 0,873 \\
Ítem 6 & 2,72 & 0,983 & 2,65 & 1,099 \\
Ítem 7 & 2,98 & 0,966 & 3,04 & 0,903 \\
Ítem 8 & 3,28 & 1,012 & 3,32 & 0,905 \\
Ítem 9 & 3,02 & 1,005 & 3,33 & 0,916 \\
Ítem 10 & 2,93 & 0,918 & 2,99 & 0,956 \\
Ítem 11 & 3,07 & 0,908 & 3,32 & 0,783 \\
Ítem 12 & 2,82 & 1,145 & 2,92 & 1,007 \\
Ítem 13 & 3,31 & 0,877 & 3,37 & 0,810 \\
Ítem 14 & 2,93 & 1,017 & 3,09 & 0,930 \\
Ítem 15 & 2,99 & 0,990 & 3,07 & 0,918 \\
\hline
\end{tabular}

Fuente: Elaboración propia.

Por otro lado, el esfuerzo del equipo directivo, en opinión del equipo docente y de las familias, ha ido dirigido, en ambos contextos, en primer y segundo lugar a: ofrecer información transparente respecto al proceso de admisión y matriculación para garantizar que llega a todos los interesados por igual (ítem 23: 3,46 y 3,59) y preocuparse por garantizar el acceso a las instalaciones y servicios del centro a todo el alumnado (ítem 35: 3,45 y 3,54). Existe una leve diferencia con respecto a la acción destacada en tercer lugar, ya que para los participantes de los centros desfavorecidos sería: trabajar para que exista un clima institucional en el que todo el alumnado es reconocido, atendido y valorado (ítem 20: 3,38), mientras que para los de centros favorecidos sería: se preocupa de que los servicios que ofrece el centro respeten las diferentes necesidades del alumnado (sensibilidades religiosas, intolerancias gastronómicas, problemas de salud...) (ítem 17: 3,50), aunque en ambos caso le sigue: tomar medidas para prevenir y evitar el absentismo escolar (ítem 24: 3,37 y 3,40).

Con respecto a las acciones que han sido menos trabajadas por ambos equipos directivos, encontramos coincidencias en relación a las dos primeras: disponer de un procedimiento de recogida de información sobre las necesidades del profesorado, alumnado y resto de personal del centro (ítem 16: 2,75, en ambos casos) y promover que los distintos miembros de la comunidad educativa participen en la evaluación de las labores de dirección (ítem 30: 2,80 y 2,79). Aunque, la tercera acción que menos ponen en práctica los equipos directivos de los centros de zonas desfavorecidas es: generar oportunidades para que todos los miembros de la comunidad educativa participen de forma efectiva en las decisiones (ítem 27: 2,95 de media) y los de zonas favorecidas: establecer mecanismos 
para impulsar la participación del alumnado en la regulación de conflictos que surgen del entorno educativo (ítem 32: 2,95 de media).

Cuadro 3. Puntuaciones medias y desviación estándar de las respuestas por ítem a la Dimensión II: Centro como comunidad inclusiva

\begin{tabular}{lcccc}
\hline & \multicolumn{2}{c}{ CoNTEXTO DeSFAVORABLE } & \multicolumn{2}{c}{ CoNTEXTO FAVORABLE } \\
\cline { 2 - 5 } & Medias & Desviación estándar & Medias & Desviación estándar \\
\hline Ítem 16 & 2,75 & 1,052 & 2,75 & 1,042 \\
Ítem 17 & 3,29 & 0,946 & 3,50 & 0,726 \\
Ítem 18 & 3,29 & 0,840 & 3,35 & 0,806 \\
Ítem 19 & 3,10 & 1,023 & 3,29 & 0,791 \\
Ítem 20 & 3,38 & 0,889 & 3,46 & 0,780 \\
Ítem 21 & 3,10 & 1,014 & 3,18 & 0,949 \\
Ítem 22 & 3,25 & 0,937 & 3,28 & 0,888 \\
Ítem 23 & 3,46 & 0,928 & 3,59 & 0,660 \\
Ítem 24 & 3,37 & 0,998 & 3,40 & 0,968 \\
Ítem 25 & 3,08 & 1,011 & 3,05 & 1,006 \\
Ítem 26 & 3,29 & 0,946 & 3,31 & 0,944 \\
Ítem 27 & 2,95 & 1,158 & 3,10 & 0,938 \\
Ítem 28 & 3,25 & 1,005 & 3,31 & 0,986 \\
Ítem 29 & 3,00 & 1,078 & 3,11 & 0,889 \\
Ítem 30 & 2,80 & 1,133 & 2,79 & 1,093 \\
Ítem 31 & 3,14 & 1,037 & 3,18 & 0,948 \\
Ítem 32 & 3,09 & 0,971 & 2,95 & 1,036 \\
Ítem 33 & 3,10 & 1,004 & 3,13 & 0,963 \\
Ítem 34 & 3,03 & 0,951 & 3,22 & 0,876 \\
Ítem 35 & 3,45 & 0,906 & 3,54 & 0,720 \\
\hline F́t & & &
\end{tabular}

Fuente: Elaboración propia.

Encontramos similitudes con respecto a las acciones que, según el equipo docente, promueven los equipos directivos de ambas zonas para que en sus centros existan Comunidades profesionales de aprendizaje (cuadro 4). Las tres primeras, en ambos casos, van dirigidas a: sensibilizar al profesorado sobre la necesidad de comunicar las situaciones de discriminación o exclusión que pueda darse en el centro (ítem 40: 3,27 y $3,58)$, se interesan por conocer la postura del profesorado en relación a la diversidad del alumnado (ítem 37: 3,16 y 3,45) y promueven la colaboración entre el profesorado para mejorar la enseñanza facilitando tiempos y espacios (ítem 36: 3,11 y 3,46).

Sin embargo, encontramos algo más de diferencias con respecto a las acciones menos implantadas por los equipos directivos de ambos contextos. Así, los directivos aún no desarrollan, en segundo lugar, de forma conveniente acciones dirigidas a proponer actividades y diseñar estrategias (seminarios, cursos, conferencias, ...) para abordar las percepciones, estereotipos, etc., del profesorado a fin de garantizar el respeto a la diversidad del alumnado y la igualdad de oportunidades (ítem 42: 2,91 y 3,10), mientras que los directivos de centros desfavorecidos no promueven, en primer lugar, espacios de reflexión entre los miembros del claustro sobre las condiciones de igualdad que ofrece el centro (ítem 39: 2,84) y los de centros favorecidos: promueven proyectos de investigación-acción en el centro con el fin de orientar procesos de mejora (ítem 38: $3,06)$. 
Destacar que, en esta dimensión, las medias de los ítems son siempre mayores en los centros pertenecientes a contextos favorecidos que la de los desfavorecidos.

Cuadro 4. Puntuaciones medias y desviación estándar de las respuestas por ítem a la Dimensión III: Comunidad profesional de aprendizaje.

\begin{tabular}{|c|c|c|c|c|}
\hline & \multicolumn{2}{|c|}{ CONTEXTO DESFAVORABLE } & \multicolumn{2}{|c|}{ CONTEXTO FAVORABLE } \\
\hline & Medias & Desviación estándar & Medias & Desviación estándar \\
\hline Ítem 36 & 3,11 & 0,903 & 3,46 & 0,685 \\
\hline Ítem 37 & 3,16 & 1,016 & 3,45 & 0,740 \\
\hline Ítem 38 & 2,99 & 1,000 & 3,06 & 0,902 \\
\hline Ítem 39 & 2,84 & 1,016 & 3,25 & 0,865 \\
\hline Ítem 40 & 3,27 & 0,944 & 3,58 & 0,643 \\
\hline Ítem 41 & 3,11 & 0,964 & 3,35 & 0,803 \\
\hline Ítem 42 & 2,91 & 1,090 & 3,10 & 0,920 \\
\hline Ítem 43 & 3,06 & 1,021 & 3,25 & 0,855 \\
\hline Ítem 44 & 2,90 & 1,006 & 3,19 & 0,903 \\
\hline Ítem 45 & 2,95 & 0,970 & 3,37 & 0,739 \\
\hline Ítem 46 & 3,06 & 1,108 & 3,29 & 0,797 \\
\hline Ítem 47 & 2,99 & 0,965 & 3,15 & 0,861 \\
\hline
\end{tabular}

Fuente: Elaboración propia.

Según se puede observar en el siguiente cuadro, todos los ítems que componen la dimensión Gestionar procesos de enseñanza-aprendizaje han sido "sustancialmente implantados" por los quipos directivos escolares de ambas zonas, ya que las medias, en todos los casos, son superiores a 3. Sin embargo, de nuevo, las medias de las acciones desarrolladas por los equipos directivos de zonas desfavorecidas, son siempre menores que las de los equipos de zonas favorecidas.

Las acciones que en primer lugar han abordado ambos equipos directivos, en opinión del equipo docente, han sido: fomentar el que se valore a las personas en función de sí mismas y no por sus características físicas, étnicas, religiosas, etc., (ítem 55: 3,55 y 3,74). Existe diferencia con respecto a la posición segunda, ya que en al caso de los equipos de zonas desfavorecidas, la acción desarrollada ha sido la de promover el que se respete, en igualdad de condiciones, las distintas creencias del alumnado (ítem 54: 3,51), mientras que en los centros de zonas favorecida ha sido la de proporcionar un trato individualizado, no discriminatorio, al alumnado para responder a sus necesidades educativas (ítem 65: 3,72). La tercera acción mayormente desarrollada en ambos contextos es la de potenciar la participación del alumnado en actividades sociales y académicas del centro Ítem 63: 3,45 y 3,68).

Por último, destacar que en las acciones menos abordadas también hay coincidencia en ambas zonas en cuanto que la menos trabajada ha sido la de promover la participación de los estudiantes en los procesos de sensibilización a través de prácticas inclusivas en el centro (ítem 66: 3,03 y 3,35). A la que le sigue, en el caso de los equipos de zonas desfavorecidas la de animar al profesorado a facilitar la construcción de la identidad del alumnado (ítem 53: 3,05) y en las zonas favorecidas: se interesa porque todo el alumnado se vea representado en los contenidos que se enseñan (ítem 52: 3,42). 
Cuadro 5. Medias y desviación estándar de las respuestas por ítem a la Dimensión IV: Gestión de los procesos de enseñanza-aprendizaje. Diferenciando por zonas

\begin{tabular}{lcccc}
\hline & \multicolumn{2}{c}{ CoNTEXTO DeSFAVORABLE } & \multicolumn{2}{c}{ CoNTEXTO FAVORABLE } \\
\cline { 2 - 5 } & Medias & Desviación estándar & Medias & Desviación estándar \\
\hline Ítem 48 & 3,30 & 0,973 & 3,52 & 0,687 \\
Ítem 49 & 3,19 & 0,957 & 3,57 & 0,569 \\
Ítem 50 & 3,30 & 0,949 & 3,55 & 0,594 \\
Ítem 51 & 3,33 & 0,919 & 3,65 & 0,519 \\
Ítem 52 & 3,23 & 0,968 & 3,42 & 0,711 \\
Ítem 53 & 3,05 & 1,183 & 3,47 & 0,677 \\
Ítem 54 & 3,51 & 0,858 & 3,71 & 0,455 \\
Ítem 55 & 3,55 & 0,934 & 3,74 & 0,454 \\
Ítem 56 & 3,41 & 0,892 & 3,65 & 0,531 \\
Ítem 57 & 3,31 & 0,876 & 3,51 & 0,687 \\
Ítem 58 & 3,32 & 0,891 & 3,55 & 0,647 \\
Ítem 59 & 3,16 & 1,016 & 3,46 & 0,714 \\
Ítem 60 & 3,06 & 1,076 & 3,45 & 0,807 \\
Ítem 61 & 3,36 & 0,833 & 3,57 & 0,635 \\
Ítem 62 & 3,26 & 0,890 & 3,52 & 0,638 \\
Ítem 63 & 3,45 & 0,843 & 3,68 & 0,480 \\
Ítem 64 & 3,28 & 1,005 & 3,46 & 0,792 \\
Ítem 65 & 3,41 & 0,942 & 3,72 & 0,541 \\
Ítem 66 & 3,03 & 1,011 & 3,35 & 0,834 \\
\hline Funte: & & &
\end{tabular}

Fuente: Elaboración propia.

En el cuadro 6 se muestran los resultados obtenidos tras el análisis diferencial mediante estadísticos no paramétricos (prueba U de Man Whitney) en función de la zona en la que están situados los centros educativos, el cual nos indica la existencia de diferencias significativas, a favor del contexto favorecido, en relación a las dimensiones III y IV (dimensiones a las que solo da respuesta el equipo docente),

Cuadro 6. Medias y diferencias significativas

\begin{tabular}{lcccc}
\hline & Total & $\begin{array}{c}\text { CONTEXTO } \\
\text { DESFAVORABLE }\end{array}$ & $\begin{array}{c}\text { CoNTEXTO } \\
\text { FAVORABLE }\end{array}$ & $\begin{array}{c}\text { Sig. U MAN- } \\
\text { WHITNEY }\end{array}$ \\
\hline Dimensión 1 & 3,03 & 2,98 & 3,05 & 0,503 \\
Dimensión 2 & 3,21 & 3,16 & 3,22 & 0,959 \\
Dimensión 3 & 3,20 & 3,03 & 3,29 & 0,018 \\
Dimensión 4 & 3,46 & 3,29 & 3,55 & 0,033 \\
Total & 3,29 & 3,14 & 3,37 & 0,099 \\
\hline
\end{tabular}

Nota: * Valor promedio. p igual o menor a 0,05.

Fuente: Elaboración propia.

En cuanto al análisis diferencial por ítems (cuadro 7), nos muestra diferencias significativas en tres de las cuatro dimensiones (I, III y IV). Así, de los 15 ítems que componen la Dimensión I: Apertura a la comunidad, existen diferencias en tres (centros en zonas favorecidas vs. desfavorecidas): promueve la colaboración con el mundo empresarial, informa a la familia de la propuesta curricular que orienta la acción educativa del centro y promueve acciones que fomentan la comunicación y participación de todas las familias en actividades educativas emprendidas dentro y fuera del centro. 
De los 12 ítems que componen la Dimensión III: El centro como Comunidad profesional de aprendizaje, existen diferencias por zonas sólo en dos: promueve la colaboración entre el profesorado para mejorar la enseñanza facilitando tiempos y espacios y promueve espacios de reflexión entre los miembros del claustro sobre las condiciones de igualdad que ofrece el centro

Por último, por lo que respecta a la Dimensión IV: Gestionar procesos de enseñanzaaprendizaje, de los 19 ítems que la componen hay diferencias significativas en 6 que muestran que los equipos directivos se preocupan de que la planificación de la enseñanza se haga de manera coordinada entre el profesorado, promueven agrupamientos heterogéneos del alumnado, disponen de un plan para dar cuenta de los logros de los estudiantes a las partes interesadas, aseguran que la evaluación se haya realizado de manera coordinada e interdisciplinar, proporcionan un trato individualizado, no discriminatorio, al alumnado para responder a sus necesidades educativas y promueven la participación de los estudiantes en los procesos de sensibilización a través de prácticas inclusivas en el centro

En todos los ítems en los que existen diferencias por zonas, estás son más positivas en los centros situados en contextos favorables, salvo en la acción relativa a "promueve la colaboración con el mundo empresarial” que está más implantada en los centros de zonas desfavorecidas.

Cuadro 7. Diferencias significativas por ítems (U Man-Whitney)

\begin{tabular}{lccccc}
\hline DimeNSIÓN 1 & SIG. & DiMENSIÓN 3 & SIG. & DIMENSIÓN 4 & SIG. \\
\hline Ítem 3 & 0,028 & Ítem 36 & 0,040 & Ítem 49 & 0,004 \\
Ítem 9 & 0,001 & Ítem 39 & 0,001 & Ítem 51 & 0,017 \\
Ítem 11 & & & Ítem 60 & 0,003 \\
& 0,010 & & & Ítem 62 & 0,048 \\
& & & Ítem 65 & 0,014 \\
& & & & Ítem 66 & 0,017 \\
\hline
\end{tabular}

Nota: p igual o menor a 0,05 .

Fuente: Elaboración propia.

\section{Discusión y conclusiones}

De acuerdo con los datos ofrecidos en el estudio, de forma general, podemos concluir que los miembros de la comunidad educativa (equipo docente y familias) de todos los centros participantes consideran que sus equipos directivos han implantado "sustancialmente" la mayor parte de las acciones que contribuyen a que sus centros sean inclusivos, agrupadas en dimensiones que hacen alusión a la Apertura a la comunidad, Centro como comunidad inclusiva, Comunidad profesional de aprendizaje y Gestionar el proceso de enseñanza-aprendizaje. Sin embargo, los equipos directivos de centros situados en contextos favorecidos lo han hecho mayormente que los de contextos desfavorecidos y, de forma más significativa, en las acciones pertenecientes a Comunidad profesional de aprendizaje y gestionar procesos de enseñanza-aprendizaje, llegando, incluso, a implantar sólo de forma "parcial" acciones relativas a la Apertura a la comunidad. Lo cual, contradice lo señalado por algunos investigadores quienes indican que no existen características diferenciales en la dirección, aunque estos se encuentren en contextos desfavorecidos (Sammons y Bakunn, 2011), o que, en contextos favorecidos y 
desfavorecidos, los equipos defienden de igual modo prácticas inclusivas (GómezHurtado, 2013; Gómez-Hurtado y Ainscow, 2014).

Un análisis más pormenorizado de las acciones llevadas a cabo por los distintos equipos directivos, también nos muestra diferencias en función de la zona, a favor de los que se encuentran en centros ubicados en zonas socio-económicamente medias y altas. Las familias y los equipos docentes de estos centros señalan que sus equipos directivos han implantado más todas las acciones que hacen que los centros sean inclusivos que los equipos de entornos desfavorecidos, salvo en aquellas que van encaminadas a promover la colaboración con el mundo empresarial de manera continuada, organizar debates abiertos a la comunidad sobre situaciones de exclusión e impulsar acciones para colaborar con otros centros educativos, conocer y compartir experiencias.

En relación a la Apertura a la comunidad, como señalara Bolhöfer (2011), los equipos directivos de ambas zonas sienten la necesidad de escuchar y tener en cuenta las demandas de las familias y de establecer lazos con la comunidad proponiendo actividades conjuntas (Precey y Mazurkiewcz, 2013), sin embargo, también en ambos contextos, los equipos atienden en menor grado las demandas del mundo laboral e impulsan acciones conjuntas con otros centros educativos, aspecto este que contradice lo señalado por Ainscow y Howes (2007) y Pamies, Senent y Essomba (2016), los cuales destacan la importancia de la participación del centro educativo con otros centros e instituciones del entorno para un buen desarrollo de la inclusión. En este sentido, aun cuando los resultados nos muestran que ambas acciones sólo están instauradas parcialmente en ambos contextos, es en los centros de zonas desfavorecidas en los que los equipos directivos las han instaurado en mayor grado.

Por lo que respecta al Centro como comunidad inclusiva, los equipos directivos de ambos contextos se muestran preocupados por propiciar confianza (Vázquez, Bernal y Liesa, 2014) y crear un entorno rico de información y transparencia (Muijs, 2003) sobre aspectos como la admisión y la matriculación, por poner a disposición de la comunidad los servicios y espacios del centro y por llevar a cabo acciones que prevengan y eviten el absentismo escolar. Cabe destacar que al igual que en la investigación desarrollada por Pamies, Senent y Essomba (2016) los directores de centros desfavorecidos prestan mayor atención a la creación de un clima en el que todo el alumnado sea reconocido, atendido y valorado propiciando una aceptación positiva del mismo.

Los datos nos muestran que aún en ambos contextos la cultura de la evaluación, así como la toma de decisiones conjunta, es uno de los aspectos menos implantados. Resultados que coinciden con los señalado por Dorczak (2013).

La necesidad de que en el centro exista una Comunidad profesional de aprendizaje se ve reflejada en acciones, por parte de los equipos directivos de ambos contextos, encaminadas a sensibilizar al profesorado sobre la necesidad de comunicar situaciones de exclusión y discriminación y a conocer su postura a hacia la diversidad, así como a promover la colaboración del mismo en los procesos de mejora de la enseñanza facilitando tiempos y espacios (Causton y Theotaris, 2017; Dorczak, 2013; Specht y Young, 2010). Sin embargo, en ambos contextos los directivos aún no desarrollan de forma conveniente acciones dirigidas a proponer actividades y diseñar estrategias (seminarios, cursos, etc.) para abordar las percepciones, estereotipos, etc., aunque los directivos de centros desfavorecidos promueven menos estos espacios de reflexión, y los 
de centros favorecidos, llevar a cabo proyectos de investigación-acción en el centro con el fin de orientar procesos de mejora.

Las acciones de ambos grupos de directivos están más centradas en la Gestión de los procesos de enseñanza-aprendizaje (Causton y Theotaris, 2017), ya que todas las acciones que componen esta dimensión han sido implantadas de forma sustancial por ellos en sus centros. Lo principal para ambos contextos es fomentar el que se valore a las personas en función de sí mismas y no por sus características físicas, étnicas, religiosas, etc., y se respete, en igualdad de condiciones, a las distintas creencias del alumnado. También se promueve una evaluación diversa y adaptada a las características del alumnado. Destacar que en las acciones menos abordadas en ambas zonas han sido la de promover la participación de los estudiantes en los procesos de sensibilización a través de prácticas inclusivas en el centro y animar al profesorado a facilitar la construcción de la identidad del alumnado e interesarse porque todo el alumnado se vea representado en los contenidos que se enseñan.

Por último, destacar que de forma significativa, las diferencias se muestran a favor de los directivos de zonas favorecidas en acciones dirigidas a: informar adecuadamente a las familias sobre la propuesta curricular que orienta la acción educativa del centro; fomentar la comunicación y participación en actividades educativas fuera y dentro del centro; promover la colaboración entre el profesorado para mejorar la enseñanza facilitando tiempos y espacios; promover espacios de reflexión sobre las condiciones de igualdad que ofrece el centro; preocuparse de que la planificación de la enseñanza se haga de manera coordinada entre el profesorado; disponer de un plan para dar cuenta de los logros de los estudiantes a las partes interesadas; asegurar que la evaluación se haya realizado de manera coordinada e interdisciplinar; proporcionar un trato individualizado $\mathrm{y}$ no discriminatorio al alumnado para responder a sus necesidades educativas y promover la participación de los estudiantes en los procesos de sensibilización a través de prácticas inclusivas en el centro.

Sólo existe diferencia, a favor del contexto desfavorecido, en la acción del equipo directivo relativa a la colaboración con el mundo laboral, debida, tal vez, a la preocupación por las comunidades de estos contextos en alcanzar más este objetivo que el puramente académico.

Las conclusiones mostradas, nos llevan a plantear la necesidad de que la administración educativa promueva la puesta en marcha de planes de mejora por parte de los equipos directivos pertenecientes a zonas desfavorecidas encaminados a la realización de acciones que promuevan la inclusión en sus centros.

Para terminar, señalar como limitación del presente estudio, la falta de significatividad estadística de la muestra. Los centros se han mostrado reacios a participar en la investigación, no por falta de interés hacia la temática, sino, por la saturación en la aplicación de cuestionarios a la que se les viene sometiendo y por el momento en que fueron aplicados (cercano a las sesiones de evaluación). Otro motivo posible, ha podido ser el elevado número de ítems del cuestionario. No obstante, los resultados que se obtienen en el presente trabajo son alentadores, lo que nos lleva a pensar en mejorar la fase de distribución del instrumento, analizando tramos temporales en los que los centros educativos se encuentren más libres de ocupaciones propias de su ámbito, para de esta forma incrementar el interés por la presente investigación y dedicar unos 
minutos para rellenar el cuestionario. Además, pensamos que se debería ampliar la muestra a nivel autonómico o nacional.

\section{Referencias}

Ainscow, M. y Booth, T. (2015). Guía para la educación inclusiva. Desarrollando el aprendizaje y la participación en los centros escolares. Recuperado de http://www.consorcio-educacioninclusiva.es

Ainscow, M. y Kaplan, I. (2005). Using evidence to encourage inclusive school development: Possibilities and challenges. Australasian Journal of Special Education, 29(2),106-116. https://doi.org/10.1080/1030011050290203

Ainscow, M. y Howes, A. (2007). Working together to improve urban secondary schools: A study of practice in one city. School Leadership and Management, 27(3), 285-300. https://doi.org/10.1080/13632430701379578

Angelides, P. (2012). Forms of leadership that promote inclusive education in Cypriot schools. Educational Management Administration $\xi^{\circ} \quad$ Leadership, $40(1), \quad$ 21-36. https://doi.org/10.1177/1741143211420614

Angelides, P., Antoniou, E. y Charalambous, C. (2010). Making sense of inclusion for leadership and schooling: A case study from Cyprus. International Journal of Leadership in Education, 13(3), 319-334. https://doi.org/10.1080/13603120902759539

Arnáiz, P., Azorín, C. y García, M. P. (2015). Evaluación de planes de mejora en centros educativos de orientación inclusiva. Profesorado: Revista de Curriculum y Formación del Profesorado, 19(3), 326-346.

Baker, S. D. (2007). Followership: The theoretical foundation of a contemporary construct. Journal of Leadership \& Organizational Studies, 14(1), 50-60. https://doi.org/10.1177/0002831207304343

Blandford, S. (2013). The impact of achievement for all on school leadership. Educational Management Administration $\quad y \quad$ Leadership, $41(1), \quad$ 45-62. https://doi.org/10.1177/1741143212462701

Bolhöfer, J. (2011). Hacia un marco de referencia en el liderazgo escolar. Recuperado de http://cfieleon.centros.educa.jcyl.es/sitio/upload/ES_Marco_de_referencia.pdf

Bolívar, A. (2010). La autonomía de los centros educativos en España. Recuperado de http://www.educacion.es/cesces/revista/presentacion_revista.htm

Causton, J. y Teoharis, G. (2017). The principal's handbook for leading inclusive schools. Baltimore, MD: Brookes Publishing Co.

Conrad, D. A. y Brown, L. I. (2011). Fostering inclusive education: Principals perspectives in Trinidad and Tobago. International Journal of Inclusive Education, 15(9), 1017-1029. https://doi.org/10.1080/13603110903490721

Diario Oficial de la Unión Europea. (2014). Conclusiones del consejo sobre liderazgo educativo eficaz (2014/C 30/O2). Recuperado de http://www.proyectoatlantida.eu/wordpress/wpcontent/uploads/2017/06/ANEXO-I-B-CONSEJO-EUROPA-2014-LIDERAZGOEDUCATIVO.pdf

Dorczak, R. (2013). Inclusion through the lens of school culture. En G. M. Ruairc, E. Ottesen y R. Precey (Eds.), Leadership for inclusive education: Vision, values and voices (pp. 47-57). Róterdam: Sense Publishers. https://doi.org/10.1007/978-94-6209-134-4_5 
Dyson, A. (2006). Beyond the school gates: Context, disadvantage and urban schools. En M. Ainscow y M. West (Eds.), Improving urban school: Leadership and collaboration (pp. 117129). Maidenhead: Open University Press.

Dyson, A., Howes, A. y Roberts, B. (2002). A systematic review of the effectiveness of schoollevel actions for promoting participation by all students. Londres: EPPI Centre, Institute of Education.

Macmillan, R. y Edmunds, A. L. (2010). Leadership for inclusion: Questions \& dilemas. En A. L. Edmunds y R. Macmillan (Eds.), Leadership for inclusion: A practical guide (pp. 1-16). Rotterdam: Sense Publishers.

Fraser, D. y Shields, C. (2010). Leaders' roles in disrupting dominant discourses and promoting inclusion. En L. Edmunds y R. Macmillan (Eds.), Leadership for inclusion: A practical guide (pp. 7-18). Rotterdam: Sense Publishers.

Gómez-Hurtado, I. (2013). Dirección y gestión de la diversidad en la escuela: Hacia un liderazgo inclusive. Revista Fuentes, 14, 61-84.

Gómez-Hurtado, I. y Ainscow, M. (2014). Hacia una escuela para todos: Liderazgo y colaboración. Investigación en la Escuela, 82, 19-30.

Iranzo, P., Tierno, J. M. y Barios, R. (2014). Autoevaluación institucional y dirección de centros $\begin{array}{lllll}\text { inclusivos. Teoría la } & \text { de } & \text { 2ducación, } & \text { 229-257. }\end{array}$ https://doi.org/10.14201/teoredu2014261229257

Klingner J. K., Arguelles M. E., Hughes M. T. y Vaughn S. (2001). Examining the school wide spread of research-based practices. Learning Disability Quarterly, 24, 221-234. https://doi.org/10.2307/1511112

Leithwood, K. y Prestine, N. (2002). Unpacking the challenges of leadership at the school and district level. Yearbook of the National Society for the Study of Education, 101(1), 42-64. https://doi.org/10.1111/j.1744-7984.2002.tboo003.x

León, M. J. (2001). La dirección de las instituciones educativas y la atención a la diversidad. En P. de Vicente Rodríguez (Coord.), Viaje al centro de la dirección de instituciones educativas (pp. 399-417). Bilbao: Ediciones Mensajero.

León, M. J. (2012). El liderazgo para y en la escuela inclusiva. Educatio Siglo XXI, 30(1), 131-157.

León, M. J. y López, M. C. (2017). La formación del líder inclusivo. En J. C. Núñez (Comp.), Perspectivas psicológica y educativa de las necesidades educativas especiales (pp. 215-225). Oviedo: SCINFOPER.

León, M. J., López, M. C., Romero, A., Crisol, E., Hinojosa, E. y Moreno, R. (2016). Liderando la educación inclusiva en centros de educación primaria y secundaria. En J. L. Bernal (Coord.), Libro de Simposios del XIV Congreso interuniversitario de organización de instituciones educativas (CIOIE) (pp. 127-153). Zaragoza: Universidad de Zaragoza.

Mac Ruairc, G. (2013). Leading inclusive schools. En F. Mac Ruairc, E. Ottesen y R. Precey (Eds.), Leadership for inclusive education. Values, vision and voices (pp. 71-80). Róterdam: Sense Publishers. https://doi.org/10.1007/978-94-6209-134-4_7

MacMillan, R. (2010). Leadership as trust and team-building for inclusive practice. En A. L. Edmunds y R. B. Macmillan (Eds.), Leadership for inclusion: A practical guide, (pp. 73-82). Róterdam: Sense Publisheres.

Muijs, D. (2003). La mejora y la eficacia de las escuelas en las zonas desfavorecidas: Resumen de resultados de investigación. REICE. Revista Iberoamericana sobre Calidad, Eficacia y Cambio en Educación, 1(2), 1-7. 
Muijs, D. (2006). New directions for school effectiveness research: Towards school effectiveness without schools. Journal of Educational Change, 7(3), 141-160. https://doi.org/10.1007/s10833-006-0002-7

Murillo, F. J. (2006). Una dirección escolar para el cambio. Del liderazgo transformacional al liderazgo distribuido. REICE. Revista Iberoamericana sobre Calidad, Eficacia y Cambio en Educación, 4(4e), 11-24.

Murillo, F. J. y Hernández-Castilla, R. (2014). Liderando escuelas justas para la justicia social. Revista Internacional de Educación para la Justicia Social (RIEJS), 3(2), 13-32.

Pamies, J., Senent, J. y Essomba, M. (2016). El liderazgo pedagógico y la implicación del profesorado como factores de éxito en centros de entornos desfavorecidos en España. RELIEVE. Revista Electrónica de Investigación y Evaluación Educativa, 22(2), 1-14.

Pont, B., Nusche, D. y Moorman, H. (2008). Mejorar el liderazgo escolar. Política y práctica. París: OCDE.

Precey, R. y Mazurkiewcz, G. (2013). Leadership for inclusion: An overview. En G. M. Ruairc, E. Ottesen y R. Precey (Eds.), Leadership for inclusive education. Values, vision and voices, (pp. 105-1 19). Róterdam: Sense Publishers.

Ryan, J. (2006). Inclusive leadership and social justice for schools. Leadership and Policy in Schools, 5(1), 3-17. https://doi.org/10.1080/15700760500483995

Sammons, P. y Bakunn, L. (2011). Effective schools, equity and teacher effectiveness: A review to the literature. Profesorado. Revista de Currículum y Formación del Profesorado, 15(3), 9-26.

Sindelar, P. T., Shearer, D. K., Yendol-Hoppey, D. y Liebert, T. W. (2006). The sustainability of inclusive school reform. Exceptional Children, 72(3), 317-331. https://doi.org/10.1177/001440290607200304

Specht, J. A. y Young, G. (2010). How administrators build schools as inclusive communities. En L. Edmunds y R. Macmillan (Eds.), Leadership for inclusion: A practical guide (pp. 65-72). Rótterdam: Sense Publishers.

Vázquez, S. Bernal, J. L. y Liesa, M. (2014). La conceptualización del liderazgo: Una aproximación desde la práctica educativa. REICE. Revista Iberoamericana sobre Calidad, Eficacia y Cambio en Educación, 12(5), 79-97.

\section{Breve CV de los autores}

\section{Ma José León}

Catedrática de Didáctica y Organización Escolar de la Universidad de Granada y directora del equipo de investigación HUM-386: FORCE Formación del Profesorado Centrada en la Escuela. Ha participado como miembro o directora en una veintena de proyectos de investigación nacionales e internacionales, entre los que destacan: "Innovación docente de calidad y mejora de la enseñanza universitaria”; "Liderazgo centrado en el aprendizaje y su impacto en la mejora: Prácticas y resultados en Secundaria..., todos ellos financiados por entidades de reconocido prestigio en el ámbito científico. Es autora de más de cincuenta artículos en revistas nacionales e internacionales y de cincuenta publicaciones e informes técnicos sobre educación inclusiva, formación del profesorado, didáctica y organización universitaria, profesionalización docente, deontología profesional. ORCID ID: 0000-0002-2776-1446. Email:mleon@ugr.es 


\section{Emilio Crisol}

Licenciado en Pedagogía por la Universidad de Granada en el año 2005 y Doctor Internacional en Ciencias de la Educación por la misma universidad en el año 2012. Premio Extraordinario de Doctorado. Miembro del grupo de investigación FORCE Formación del Profesorado Centrada en la Escuela desde el año 2005. En la actualidad es Profesor Ayudante Doctor en la Facultad de Ciencias de la Educación de la Universidad de Granada en el Departamento de Didáctica y Organización Escolar. Sus líneas de investigación son entre otras, la innovación; metodología didáctica, currículo y formación; la logopedia escolar, Educación Especial, etc. Actualmente participa en el proyecto de investigación: "Identidad de la dirección escolar: Liderazgo, formación y profesionalización" (Ref. EDU2016-78191-P). ORCID ID: 0000-0002-3091-6699. Email: ecrisol@ugr.es

\section{Rubén Moreno Arrebola}

Graduado en Educación Primaria con mención en educación física en 2014. En 2016 realizó el Máster en Investigación e Innovación en Currículum y Formación. Actualmente se encuentra en $2^{\circ}$ año de doctorado y trabajando como maestro especialista de educación física. En cuanto a investigación, es autor principal de 4 artículos y como coautor en 2 capítulos de libro y en 5 artículos. Ha participado como autor y coautor de 12 ponencias en congresos, (simposios, comunicaciones, posters). Como formación adicional, obtuvo en 2015 el título federativo de "técnico en primeros auxilios, salvamento y socorrismo acuático", el título de "monitor de turismo activo y deporte de aventura" y el diploma de soporte vital básico y desfibrilación semiautomática. Ha sido becario ICARO en la Universidad de Granada (2016 -2017) en el Vicedecanato de Prácticum. ORCID ID: 0000-0002-1270-393X. Email: rubenmorenoarrebola@ugr.es 